\title{
Efeito da associação de furosemida e fenilbutazona sobre variáveis hidroeletrolíticas de cavalos antes e após a corrida
}

\author{
[The effect of furosemide and phenylbutazone association on the fluid and electrolyte characteristics of \\ horses before and after a race] \\ A.S. Camargos ${ }^{1}$, A.P.D. Costa ${ }^{2}$, C.B. Carvalho ${ }^{2}$, R.C. Guidi ${ }^{2}$, C.R. Quirino ${ }^{2}$, \\ A.M.B. Batista ${ }^{2}$, J.A. Machado ${ }^{2}$ \\ ${ }^{1}$ Aluna de pós-graduação - FMVZ - UNESP Botucatu - Botucatu, SP \\ ${ }^{2}$ Universidade Estadual do Norte Fluminense Darcy Ribeiro \\ Av. Alberto Lamego, 2000 \\ 28013-600 - Campos dos Goytacazes, RJ
}

\begin{abstract}
RESUMO
Verificaram-se os efeitos da associação de furosemida e fenilbutazona sobre variáveis hidroeletrolíticas de cavalos antes e após a corrida. Dezenove equinos foram distribuídos em três grupos, de acordo com os protocolos de tratamento. O primeiro grupo, de cinco animais, não recebeu medicação (grupo-controle); o segundo grupo, de sete animais, foi tratado com furosemida, na dose de $1 \mathrm{mg} / \mathrm{kg}$, por via intramuscular, até quatro horas antes do páreo; o terceiro, de sete animais, recebeu furosemida, por via intramuscular, e fenilbutazona, por via intravenosa, nas doses de 1,0 e 4,4mg/kg, respectivamente, até quatro horas antes da corrida. Amostras de sangue foram colhidas antes, imediatamente após e duas horas após o páreo, para avaliação da osmolalidade plasmática e das concentrações plasmáticas de sódio, potássio e cloreto. A utilização de furosemida e da associação furosemida e fenilbutazona até $4 \mathrm{~h}$ antes dos páreos nas dosagens descritas alterou $(\mathrm{P}<0,05)$ a osmolalidade plasmática dos equinos, mas não alterou $(\mathrm{P}>0,05)$ as concentrações de sódio, potássio e cloreto. Os páreos alteraram de forma fisiológica a osmolalidade plasmática e a concentração sanguínea de $\mathrm{K}^{+}$devido ao exercício de alta intensidade.
\end{abstract}

Palavras-chave: equino atleta, eletrólitos, osmolalidade plasmática, diurético

\begin{abstract}
The objective of this study was to verify the effects of furosemide and phenylbutazone association on fluid and electrolyte balance characteristics of horses before and after a race. Nineteen horses were divided into three groups according to treatment protocols. The first group (five animals - control) was not medicated. A second group (seven animals) was treated with furosemide (1mg/kg, intramuscular up to four hours before the race). A third group (seven animals) received furosemide (1mg/kg) and phenylbutazone $(4.4 \mathrm{mg} / \mathrm{kg})$, both intramuscular, up to four hours before race. Blood samples were collected before, immediately after and two hours after a race to evaluate the plasma osmolality and sodium, potassium and chloride concentrations. The use of furosemide and furosemide plus phenylbutazone up to four hours before the race altered $(P<0.05)$ the plasma osmolality but did not change $(P>0.05)$ the sodium, potassium and chloride concentrations. It was not possible to determine an antagonist effect of phenylbutazone on furosemide, based on fluid and electrolyte balance. Due to the high intensity exercise, the increase in plasma osmolality and potassium concentration was attributed to the race effect.
\end{abstract}

Keywords: athletic horses, electrolytes, plasma osmolality, diuretic

Recebido em 23 de março de 2011

Aceito em 6 de junho de 2011

E-mail: aline_camargos@hotmail.com 


\section{INTRODUÇÃO}

Os equilíbrios hídrico e eletrolítico são fundamentais para o funcionamento do organismo (Cohen et al., 1993). O equilíbrio hídrico consiste na manutenção e no controle da composição dos líquidos corporais, e o equilíbrio eletrolítico na manutenção da concentração normal de íons nos diversos compartimentos (Costa, 2003). Os principais eletrólitos no equilíbrio hidroeletrolítico são $\mathrm{Na}+\mathrm{K}+$ e $\mathrm{Cl}-$, cujas concentrações plasmáticas permitem avaliar o estado de hidratação do animal (Johnson, 1998; Costa, 2003).

Um importante indicativo do equilíbrio hídrico do organismo é o equilíbrio existente entre as osmolalidades dos compartimentos intra e extracelular, denominado osmolalidade plasmática, que se reflete principalmente pelas concentrações de $\mathrm{Na}^{+}$e $\mathrm{Cl}^{-}$(Carlson e Jones, 1999). A osmolalidade plasmática é mantida sob rigorosa regulação pelo organismo, e sua variação fisiológica não ultrapassa o limite de $2 \%$ em relação ao seu valor de normalidade (Johnson, 1998).

$\mathrm{O} \mathrm{Na}^{+}$, junto com o $\mathrm{K}^{+}$, é responsável pelo gradiente osmótico e pela movimentação da água entre os compartimentos. $\mathrm{O} \mathrm{K}^{+}$é o principal cátion do compartimento intracelular. Portanto, a determinação da sua concentração plasmática pode não refletir sua quantidade corporal total (Muir et al., 1988). Já o $\mathrm{Cl}^{-}$está relacionado ao equilíbrio dos íons $\mathrm{Na}^{+}$no compartimento extracelular (Johnson, 1998).

Durante o exercício intenso de curta duração, as concentrações sanguíneas de $\mathrm{Na}^{+}$e $\mathrm{Cl}^{-}$ apresentam diferenças mínimas, pois sua quantidade no plasma diminui simultaneamente à diminuição do volume de fluidos corporais. Porém, quando é ativado o mecanismo anaeróbico para obtenção de energia, pode ocorrer diminuição significativa da concentração de $\mathrm{Cl}^{-}$diferente do $\mathrm{K}^{+}$, que apresenta aumento considerável durante $\mathrm{O}$ exercício intenso (Kingston, 2004). No entanto, esses efeitos sobre o equilíbrio hidroeletrolítico podem ser acentuados quando se associa o exercício à administração de alguns medicamentos, como a furosemida. A furosemida é um diurético da alça de Henle, utilizado para aumentar a excreção de fluidos através da urina, por impedir a absorção de água pela inibição da reabsorção primária de $\mathrm{Cl}^{-}$, e secundária de $\mathrm{Na}^{+}$na alça de Henle. Este diurético também aumenta a excreção de eletrólitos pela urina, o que acarreta diminuição nas concentrações sanguíneas de $\mathrm{K}^{+}, \mathrm{Cl}^{-}, \mathrm{Ca}^{++} \mathrm{e}$ $\mathrm{H}^{+}$. Há diminuição rápida e profunda do plasma sanguíneo após a sua administração (Hinchcliff, 2004).

A furosemida é largamente utilizada em equinos, sendo o diurético de eleição no tratamento profilático da hemorragia pulmonar induzida pelo exercício (HPIE) em cavalos de corrida (Moran e Araya, 2003). A HPIE apresenta alta prevalência e acomete mais de $60 \%$ dos cavalos de corrida (Hinchcliff, 2004). Está associada à ruptura dos capilares pulmonares, devido às altas pressões na vasculatura pulmonar durante $\mathrm{o}$ exercício (Harkins, 1997). Não há ainda um tratamento eficaz, sendo a furosemida indicada como profilática (Derksen, 1996), pois alivia as pressões de sístole do átrio direito e arteriopulmonar, pela diminuição do volume sanguíneo corrente (Radostits et al., 2002). O Jockey Clube Brasileiro regulamenta a administração da dose mínima de 100mg e máxima de $250 \mathrm{mg}$, aplicada até quatro horas antes do horário do páreo (Jockey..., 2001).

Outro tipo de medicamento utilizado rotineiramente em cavalos de corrida é a fenilbutazona, o anti-inflamatório não esteroide mais largamente empregado na clínica equina. A fenilbutazona é usada por sua atividade antiinflamatória, analgésica e antipirética no tratamento de várias formas de claudicação (Booth e McDonald, 1992). É administrada com doses entre 2,2 e $8,8 \mathrm{mg} / \mathrm{kg}$, porém a dose de $4,4 \mathrm{mg} / \mathrm{kg}$ é a mais utilizada em equinos (Mills $e t$ al., 1996). Esse anti-inflamatório inibe a atividade da cicloxigenase e a subsequente atividade de conversão de ácido araquidônico em metabólitos de prostaglandina (Hinchcliff, 1995).

Aparentemente, não ocorre retenção de eletrólitos e água no equino após a administração de fenilbutazona. Entretanto, quando associada à furosemida, a fenilbutazona antagoniza os efeitos dessa aumentando o fluxo sanguíneo renal, inibindo a sua síntese de prostaglandina e diminuindo a excreção renal do diurético (Booth e McDonald, 1992), além de minimizar a diminuição das pressões arteriopulmonar e do átrio direito, induzidas pela furosemida. Esse 
anti-inflamatório diminui o efeito diurético da furosemida, mas não o elimina (Hinchcliff, 1995). Alguns estudos afirmaram que a fenilbutazona em altas doses pode diminuir os efeitos cardiovasculares da furosemida (Manohar et al., 1998), inibindo os seus efeitos hemodinâmicos, porém sem influenciar a sua excreção (Dyke et al., 1999). Já outros estudos não obtiveram alterações significativas no organismo quando administraram ambos (Hinchcliff, 2004).

O presente estudo teve como objetivo verificar os efeitos da associação de furosemida e fenilbutazona sobre variáveis hidroeletrolíticas de cavalos antes e após a corrida.

\section{MATERIAL E MÉTODOS}

Foram coletadas amostras de sangue de 19 cavalos da raça Puro Sangue Inglês, com idade entre três e cinco anos, machos e fêmeas, durante páreos vespertinos de 1300 metros em pista de areia no Jockey Club de Campos, em Campos dos Goytacazes, RJ. No dia das provas, a temperatura ambiente média era $22,6^{\circ} \mathrm{C}$, a umidade relativa do ar média de $82,6 \%$, a radiação solar de $256 \mathrm{Wn}^{-2}$ e a velocidade do vento a $2 \mathrm{~m}$ de altura de $2,0 \mathrm{~ms}^{-1}$ (Spectrum, EUA).

A escolha dos animais foi aleatória, considerando-se apenas a notificação do proprietário de medicação dos animais com furosemida e/ou fenilbutazona, procedimento este que era permitido até quatro horas antes dos páreos pelo Jockey Club de Campos na época do experimento. Os animais tratados já haviam recebido a mesma medicação no páreo anterior 3-4 dias antes. Foram incluídos nos grupos experimentais apenas os animais que não apresentaram distúrbios ao exame clínico.

Os animais foram distribuídos em três grupos, de acordo com o protocolo de medicação. O primeiro grupo (G0), com cinco animais, não recebeu medicação (grupo-controle); o segundo grupo (G1), formado por sete animais, foi tratado com furosemida, na dose de $1,0 \mathrm{mg} / \mathrm{kg}$ por via intramuscular, até quatro horas antes do páreo; o terceiro grupo (G2), com sete animais, recebeu furosemida por via intramuscular e fenilbutazona por via intravenosa, nas doses de 1,0 e 4,4mg/kg, respectivamente, até quatro horas antes da corrida.

As coletas de sangue foram realizadas em três momentos: uma hora antes dos páreos (M1), imediatamente após (M2) e duas horas após o páreo (M3). A coleta no momento 0 , anterior à aplicação dos tratamentos, não foi possível devido a restrições dos proprietários. As amostras foram coletadas na veia jugular utilizando-se sistema a vácuo e agulha descartável $25 \times 0,8 \mathrm{~mm}$. Durante a coleta, foram acondicionadas em dois tubos: sem anticoagulante ou com EDTA. Após a coleta, as amostras foram imediatamente centrifugadas a $1372 \mathrm{~g}$ por 10 minutos para separação do soro ou plasma. Em seguida, foram acondicionadas em tubos criogênicos do tipo Eppendorf $\mathrm{e}$ congeladas a $-20^{\circ} \mathrm{C}$.

As amostras colhidas sem anticoagulante foram destinadas à determinação de osmolalidade plasmática, utilizando-se osmômetro e método de crioscopia (Osmomett-A, EUA), e das concentrações de $\mathrm{Na}^{+}$e $\mathrm{K}^{+}$, por meio de fotômetro de chama (Benfer BFC 150®, Brasil). A concentração de $\mathrm{Cl}^{-}$foi determinada utilizando-se kits reagentes fabricados pela Analisa Diagnóstica ${ }^{\circledR}$, em um espectrofotômetro (Microlab 200 Merck®, Germany), em amostras obtidas no tubo com EDTA.

Para a análise estatística, as variáveis osmolalidade plasmática, $\mathrm{Na}^{+}, \mathrm{K}^{+}$e $\mathrm{Cl}^{-}$foram avaliadas utilizando-se o programa SAS (SAS, 1996), em modelo de delineamento inteiramente ao acaso. Foram determinados, para cada variável, a consistência dos dados, a estatística descritiva, a análise de variância $(a=0,05)$ e o teste Tukey para comparação de médias. Foram avaliados os efeitos dos tratamentos, dos momentos e a interação tratamento $\mathrm{X}$ momento. No caso da osmolalidade plasmática, foi observada diferença significativa na interação tratamento $\mathrm{X}$ momento, sendo então realizado o desdobramento da análise, avaliando-se os efeitos dos momentos dentro dos tratamentos e dos tratamentos dentro dos momentos pelo teste Tukey. 


\section{RESULTADOS E DISCUSSÃO}

Houve diferença significativa $(\mathrm{P}<0,05)$ entre os tratamentos (grupos) apenas para osmolalidade plasmática (Tab. 1). De modo geral, o tratamento com furosemida não alterou esta variável; por outro lado, a associação de furosemida e fenilbutazona induziu valores de osmolalidade plasmática abaixo dos observados nos demais tratamentos. Não há relatos na literatura consultada sobre alteração na osmolalidade plasmática induzida pela associação de furosemida e fenilbutazona na dosagem descrita neste estudo.

$\mathrm{Na}$ osmolalidade plasmática, foram observadas diferenças $(\mathrm{P}<0,05)$ entre os momentos e o efeito da interação tratamento $\mathrm{X}$ momento (Tab. 2).
Observou-se que, no repouso, apenas os valores de osmolalidade plasmática dos equinos do grupo-controle apresentaram valores considerados normais, entre 270 e $300 \mathrm{mOsm} / \mathrm{kg}$, segundo Feldman e Rosenberg (1981); Brownlow e Hutchins (1982) e Genetzky et al. (1987). A furosemida e a associação furosemida e fenilbutazona, nos tratamentos 1 e 2, propiciaram diminuição da osmolalidade plasmática antes da corrida. Após o páreo, no entanto, somente os animais do G2 continuaram com valores mais baixos. Estes resultados sugerem que a fenilbutazona pode ter exercido efeito aditivo quando associada à furosemida, sugerindo prolongar o seu efeito sobre a osmolalidade plasmática. Desse modo, não foi possível verificar efeito antagonista da fenilbutazona sobre a furosemida.

Tabela 1. Osmolalidade plasmática e concentrações sanguíneas de sódio, potássio e cloreto, após a aplicação de furosemida e fenilbutazona, em cavalos PSI avaliados antes e após páreos de 1300m (média \pm desvio-padrão)

\begin{tabular}{lcccccc}
\hline \multirow{2}{*}{ Variável } & \multicolumn{3}{c}{ Tratamento } & \multicolumn{3}{c}{ Momentos } \\
\cline { 2 - 7 } & G0 & G1 & G2 & M1 & M2 & M3 \\
\hline \multirow{2}{*}{ Osm. plasm. (mOsm/kg) } & $284,65 \pm$ & $285,05 \pm$ & $275,00 \pm$ & $267,55 \pm$ & $314,87 \pm$ & $270,31 \pm$ \\
& $24,58 \mathrm{~A}$ & $25,42 \mathrm{~A}$ & $17,17 \mathrm{~B}$ & $10,18 \mathrm{~B}$ & $10,18 \mathrm{~A}$ & $6,24 \mathrm{~A}$ \\
$\mathrm{Na}^{+}(\mathrm{mEq} / \mathrm{L})$ & $135.19 \pm$ & $136.73 \pm$ & $135.30 \pm$ & $133.37 \pm$ & $135.53 \pm$ & $138.17 \pm$ \\
& $3.98 \mathrm{~A}$ & $7.77 \mathrm{~A}$ & $12.73 \mathrm{~A}$ & $7.53 \mathrm{~A}$ & $8.65 \mathrm{~A}$ & 9.95 \\
$\mathrm{~K}^{+}(\mathrm{mEq} / \mathrm{L})$ & $3.33 \pm$ & $3.15 \pm$ & $3.10 \pm$ & $2.96 \pm$ & $3.62 \pm$ & $3.01 \pm$ \\
& $0.62 \mathrm{~A}$ & $0.65 \mathrm{~A}$ & $0.44 \mathrm{~A}$ & $0.45 \mathrm{~B}$ & $0.58 \mathrm{~A}$ & $0.43 \mathrm{~B}$ \\
$\mathrm{Cl}^{-}(\mathrm{mEq} / \mathrm{L})$ & $97.00 \pm$ & $87.47 \pm$ & $84.05 \pm$ & $88.68 \pm$ & $91.47 \pm$ & $89.00 \pm$ \\
& $30.73 \mathrm{~A}$ & $23.05 \mathrm{~A}$ & $31.57 \mathrm{~A}$ & 33.32 & 24.68 & $30.80 \mathrm{~A}$ \\
\hline
\end{tabular}

Osmolalidade Plasmática.

G0 - grupo-controle (sem medicação); G1 - grupo que recebeu furosemida; G2 - grupo que recebeu furosemida associada a fenilbutazona.

Momentos: M1 - 1 hora antes do páreo; M2 - imediatamente após o páreo; M3 - duas horas após o páreo.

Médias seguidas por letras distintas, na mesma linha, diferem entre si $(\alpha=0,05)$.

Tabela 2. Média e desvio-padrão de osmolalidade plasmática ( $\mathrm{mOsm} / \mathrm{kg}$ ) em cavalos do corrida tratados com furosemida e fenilbutazona, segundo o momento de avaliação

\begin{tabular}{|c|c|c|c|}
\hline & \multicolumn{3}{|c|}{ Tratamentos } \\
\hline Grupo & M1 & M2 & M3 \\
\hline G0 & $271,14 \pm 15,73 \mathrm{Aa}$ & $316,83 \pm 14,36 \mathrm{Aa}$ & $270,57 \pm 3,87 \mathrm{Aa}$ \\
\hline G1 & $265,28 \pm 6,07 \mathrm{Ab}$ & $318,67 \pm 4,93 \mathrm{Aa}$ & $272,40 \pm 9,07 \mathrm{Aa}$ \\
\hline $\mathrm{G} 2$ & $266,00 \pm 4,94 \mathrm{Aa}$ & $303,33 \pm 9,45 \mathrm{Aa}$ & $267,25 \pm 5,91 \mathrm{Aa}$ \\
\hline
\end{tabular}

G0 - grupo controle (sem medicação); G1 - grupo que recebeu furosemida; G2 - grupo que recebeu furosemida e fenilbutazona; M1- 1h antes do páreo; M2 - imediatamente após o páreo, M3 - 2h após o páreo.

Quando avaliado o efeito de tratamento dentro de cada momento, médias seguidas por letras distintas maiúsculas, na linha, diferem entre si $(\alpha=0,05)$.

Quando avaliado o efeito de momento dentro de cada tratamento, médias seguidas por letras distintas minúsculas, na coluna diferiram entre si $(\alpha=0,05)$. 
Imediatamente após os páreos, observou-se aumento na osmolalidade plasmática em todos os grupos, mas este aumento foi menos evidente no grupo tratado com furosemida e fenilbutazona. Ocorreu aumento da tonicidade do plasma neste momento associada à discreta desidratação hipertônica, estado que confirma os resultados observados. Os valores de osmolalidade plasmática duas horas após os páreos foram considerados similares aos observados précorrida.

Não houve diferença significativa $(\mathrm{P}>0,05)$ entre os tratamentos, quando avaliadas as concentrações sanguíneas de $\mathrm{Na}^{+}, \mathrm{K}^{+}$e $\mathrm{Cl}^{-}$. Isso se deve, provavelmente, ao intervalo obtido entre a administração das drogas e a competição (acima de 4h). Aparentemente, esse intervalo regulamentado para a utilização da furosemida proporcionou tempo suficiente para que o organismo restabelecesse o equilíbrio eletrolítico. Dyke et al. (1999) também não observaram alterações na excreção de eletrólitos induzidas após utilização de furosemida e fenilbutazona, enquanto Carlson e Jones (1999) relataram diminuição da concentração de $\mathrm{Cl}^{-}$ após a administração de furosemida em cavalos de corrida. Não foi possível determinar o efeito antagonista da fenilbutazona sobre a furosemida, considerando-se o equilíbrio eletrolítico.

Quanto ao efeito dos momentos sobre as concentrações sanguíneas de $\mathrm{Na}^{+}, \mathrm{K}^{+}$e $\mathrm{Cl}^{-}$, apenas a concentração de $\mathrm{K}^{+}$apresentou diferença significativa $(\mathrm{P}<0,05)$ entre momentos. Ocorreu aumento médio de $22,3 \%$ na concentração de $\mathrm{K}^{+}$imediatamente após o páreo (Tab. 1). Este aumento confirma os achados de Walter e Lindinger (2004), que observaram aumento na concentração sanguíneas de $\mathrm{K}^{+}$nos primeiros minutos após o páreo.

$\mathrm{O}$ aumento da concentração de $\mathrm{K}^{+}$, neste estudo, pode ser atribuído à liberação de $\mathrm{K}^{+}$e lactato pelos músculos, que ocorre durante exercícios de alta intensidade e curta distância, pela ativação da glicólise anaeróbica (McKeever, 2004). O aumento da produção de lactato contribui para um quadro de acidose metabólica, que leva à hipercalemia pela saída do $\mathrm{K}^{+}$do fluido intracelular para o extracelular - substituição do $\mathrm{K}^{+}$pelo hidrogênio no compartimento intracelular; o $\mathrm{K}^{+}$liberado cai na corrente sanguínea e ocorre aumento da concentração plasmática desse eletrólito. Segundo McKeever (2004), os valores de $\mathrm{Na}^{+}$e $\mathrm{Cl}^{-}$podem ou não sofrer alterações, e isso vai depender da distância ou do tempo total do exercício.

Resultados diferentes foram descritos por Martins et al. (2003), Fernandes e Larsson (2000) e Ribeiro et al. (2004) após participação em prova de enduro, no qual a concentração sanguínea de $\mathrm{K}^{+}$diminuiu. Isto ocorre, pois, em exercícios de baixa intensidade e longa duração, há sudorese excessiva associada à perda significativa de $\mathrm{K}^{+}$(Martins et al., 2003). Duas horas após o páreo, a concentração sanguínea de $\mathrm{K}^{+}$já havia retornado ao valor similar ao anterior à prova.

\section{CONCLUSÕES}

A utilização de furosemida e da associação furosemida e fenilbutazona até $4 \mathrm{~h}$ antes do páreo nas dosagens descritas alterou a osmolalidade plasmática dos equinos pela ação diurética desses medicamentos, mas não alterou as concentrações de sódio, potássio e cloreto. Os páreos alteraram de forma fisiológica a osmolalidade plasmática e a concentração sanguínea de $\mathrm{K}^{+}$devido ao exercício de alta intensidade.

\section{REFERÊNCIAS BIBLIOGRÁFICAS}

BOOTH, N.H.; McDONALD, L.E. Farmacologia e terapêutica em veterinária. Rio de Janeiro: Guanabara Koogan, 1992. 1575p.

BROWNLOW, M.A.; HUTCHINS, D.R. The concept of osmolality: Its use in the evaluation of "dehydration" in the horse. Equine Vet. J., v.14, p.106-110, 1982.

CARLSON, G.P.; JONES, J.H. Effects of frusemide on electrolyte and acid-base balance during exercise. Equine Vet. J., v.30, p.370-374, 1999.

COHEN, N.D.; ROUSSEL, A.J.; LUMSDEN, J.H. et al. Alterations of fluid and electrolyte balance in thoroughbred racehorses following strenuous exercise during training. Can. J. Vet. Res., v.57, p.9-13, 1993.

COSTA, A.P. Determinação dos efeitos da desidratação experimental nos equilíbrios hidroeletrolítico e ácido-base de equinos. 2003. 57f. Dissertação (Mestrado em Produção Animal) Universidade Estadual do Norte Fluminense Darcy Ribeiro, Campos dos Goytacazes. 
DERKSEN, F.J. Update on Exercise-Induced Pulmonary Hemorrhage. Equine Athlete, v.9, p.16-18, 1996.

DYKE，T.M.; HINCHCLIFF，K.W.; SAMS, R.A. Attenuation by phenylbutazone of the renal effects and excretion of frusemide in horses. Equine Vet. J., v.31, p.289-95, 1999.

FELDMAN, B.F.; ROSENBERG, D.P. Clinical use of anion and osmol gap in veterinary medicine. J. Am. Vet. Med. Assoc., v.178, p.396398, 1981.

FERNANDES，W.R.; LARSSON， M.H.M.A. Alterações nas concentrações séricas de glicose, sódio, potássio, ureia e creatinina em equinos submetidos a provas de enduro de $30 \mathrm{~km}$ de velocidade controlada. Cienc. Rural, v.30, p.393398, 2000.

GENETZKY, R.; LOPARCO, F.V.; LEDET, A.E. Clinical pathologic alterations in horses during a water deprivation test. Am. J. Vet. Res., v.48, p.1007-1011, 1987.

HARKINS, J.D. Exercise-Induced Pulmonary Hemorrhage: A review of the etiology and pathogenesis. Equine Pract., v.19, p.22-28, 1997.

HINCHCLIFF, K.W. Drug effects on performance. In: HINCHCLIFF, K.W. et al. Equine sports medicine and surgery: basic and clinical sciences of the equine athlete. Philadelphia: Saunders, 2004. p.1268-1291.

HINCHCLIFF, K.W. Pharmacologic interaction of furosemide and phenylbutazone in horses. Am. J. Vet. Res., v.56, p.1206-12, 1995.

JOCKEY Club Brasileiro. Código nacional do jockey club Brasileiro. 2001. Disponível em: http://www.jcb.com.br/asapostas/apendicecodigo nacional.doc. Acessado em 27 jan. 2010.

JOHNSON, P.J. Physiology of body fluids in the horse. Vet. Clin. North Am., v.14, p.1-22, 1998.

KINGSTON, J.K. Hematologic and serum biochemical responses to exercise and training. In: HINCHCLIFF, K.W. et al. Equine sports medicine and sugery: basic and clinical sciences of the equine athlete. Philadelphia: Saunders, 2004. p.939-948.
MANOHAR, M.; GOETZ, T. E.; SULLIVAN, E. et al. Pulmonary vascular pressures of strenuously exercising thoroughbred horses after administration of phenylbutazone and frusemide. Equine Vet. J., v.30, p.158-162, 1998.

MARTINS, C.B.; OROZCO, C.A.G.; DANGELIS, F.H.F. et al. Avaliação de variáveis bioquímicas de equinos antes e após participação em prova de enduro. Rev. Univ. Rural, v.23, p.175-176, 2003.

MCKEEVER, K.H. Body fluids and electrolytes: responses to exercise and training. In: HINCHCLIFF, K.W. et al. Equine sports medicine and surgery: basic and clinical sciences of the equine athlete. Philadelphia: Saunders, 2004. p.853-871.

MILLS, P.C.; Ng, J.C.; AUER, D.E. The effect of inflammation on the disposition of phenylbutazone in thoroughbred horses. J. Vet. Pharmacol. Ther., v.19, p.475-81, 1996.

MORAN, G.; ARAYA, O. Hemorragia pulmonar inducida por el ejercicio en el cabalo: una revisión. Arch. Med. Vet., v.35, p.1-12, 2003.

MUIR, W.W.; KHON, C.W.; SAMS, R. Effects of furosemide on plasma volume and extracellular fluid volume in horses. Am. J. Vet. Res., v.39, p.1688-1691, 1988.

RADOSTITS, O.M. Clínica veterinária: um tratado de doenças dos bovinos, ovinos, suínos, caprinos e equinos. Rio de Janeiro: Guanabara Koogan, 2002. 1737 p.

RIBEIRO, C.R.; MARTINS, E.A.N.; RIBAS, J.A.S. et al. Avaliação de constituintes séricos em equinos e muares submetidos à prova de resistência de $76 \mathrm{~km}$, no Pantanal do Mato Grosso, Brasil. Cienc. Rural, v.34, p.155-174, 2004.

SAS. User's Guide. SAS Inst. Inc. Cary, NC, 1996.

WALTER, A.; LINDINGER, M.I. Time course and magnitude of fluid and electrolyte shifts during recovery from high-intensity exercise in Standardbred racehorses. Equine comp. exerc. physiol., v.2, p.77-87, 2004. 